\title{
miR-573 regulates melanoma progression by targeting the melanoma cell adhesion molecule
}

\author{
HE-FEI WANG ${ }^{1}$, HONG CHEN $^{2}$, MIN-WANG MA $^{3}$, JI-AN WANG $^{4}$, TING-TING TANG ${ }^{5}$, \\ LAI-SHENG NI ${ }^{6}$, JIN-LING YU ${ }^{7}$, YU-ZHEN LI ${ }^{1}$ and BING-XUE BAI ${ }^{1}$
}

\begin{abstract}
${ }^{1}$ Department of Dermatology, The Second Affiliated Hospital of Harbin Medical University, Harbin, Heilongjiang 150086;
${ }^{2}$ Department of Pediatrics, The First Affiliated Hospital of Heilongiang University of Chinese Medicine, Harbin, Heilongjiang 150040; ${ }^{3}$ Department of Ophthalmology, The Affiliated Hospital of Medical College of Chinese People's Armed Police Forces, Tianjin 300162; ${ }^{4}$ Obstetrics and Gynecology Hospital, Harbin, Heilongjiang 150300; ${ }^{5}$ Orthopedic Surgery of

Shanghai Ninth People's Hospital Affiliated to Shanghai Jiao Tong University School of Medicine, Shanghai 200025;

${ }^{6}$ Normal Surgical Department of the People's Hospital of Acheng City, Harbin, Heilongjiang 150300;

${ }^{7}$ Pathology of General Hospital of Jixi Mining Group, Jixi, Heilongjiang 158100, P.R. China
\end{abstract}

Received January 16, 2013; Accepted April 16, 2013

DOI: 10.3892/or.2013.2451

\begin{abstract}
Melanoma is a malignant tumor of the melanocytes. microRNAs (miRNAs) are emerging as important regulators of cancer-related processes. A thorough understanding of miRNAs in melanoma progression is important for developing new therapeutic targets. miRNA expression was detected by quantitative PCR. In vitro, MTT assay, colony formation assay, invasion assay and flow cytometry analysis were performed to test the effect of miR-573 on melanoma cells. The effect of miR-573 in vivo was validated using a murine xenograft model. Using quantitative PCR, we found that the expression levels of miR-573 were lower in melanoma tissues and cell lines compared to normal skin tissues. miR-573 upregulation inhibited melanoma cell proliferation and invasion, and overexpression of melanoma cell adhesion molecule (MCAM) could alleviate the effect of miR-573 on melanoma cells. In vivo, miR-573 overexpression groups showed lower rates of tumor growth compared with the control group. In conclusion, our results demonstrate that the elevated MCAM expression due to miR-573 reduction is essential in melanoma initiation and progression.
\end{abstract}

\section{Introduction}

Melanoma is a malignant tumor of the melanocytes. Statistical data show that melanoma is the cause of approximately $75 \%$

Correspondence to: Professor Yu-Zhen Li or Dr Bing-Xue Bai, Department of Dermatology, The Second Affiliated Hospital of Harbin Medical University, 246 Xuefu Road, Nangang, Harbin, Heilongjiang 150086, P.R. China

E-mail: liyuzhen4@gmail.com

E-mail: bingxuebai669@163.com

Key words: melanoma, microRNA, miR-573, melanoma cell adhesion molecule of all skin cancer-related deaths. In European countries, there were approximately 60,000 melanoma cases leading to 13,000 deaths in 2006 (1). Elucidating the detailed mechanisms of melanoma has been a challenging task for researchers.

The melanoma cell adhesion molecule (MCAM), also known as CD146 or MUC18, is a membrane calcium-independent glycoprotein adhesion molecule first identified in melanoma (2). MCAM is abnormally expressed in other tumor tissues, including prostate cancer (3), breast cancer (4), and non-small cell lung cancer (5). MCAM is also a characteristic antigen that distinguishes malignant melanoma from benign or borderline melanoma. To date, the upstream regulation of MCAM remains largely unknown. Thus, we investigated the factors that directly regulate MCAM.

microRNAs (miRNAs) are short non-coding RNAs (18-22 nt) that can inhibit gene expression at the post-transcription level. Following processing by Drosha and Dicer, mature miRNAs are incorporated into the RNA-induced silencing complex (RISC), bind to the $3^{\prime}$ untranslated region (3'UTR) of the target mRNAs and inhibit their expression $(6,7)$. Due to their widespread regulation on protein-coding genes, miRNAs have various physiological and pathological functions. Accumulating evidence demonstrates the relationship between miRNAs and carcinogenesis $(8,9)$. Several miRNA-regulated molecular pathways are involved in the pathogenesis of malignant melanoma, including the RAS-RAF-MEK-ERK, p16(INK4A)-CDK4-RB, PIK3-AKT, and MITF pathways (10).

In the present study, we found that miR-573 was downregulated in melanoma tissues and cell lines compared to the normal skin tissues. The overexpression of miR-573 in vitro suppressed the proliferation of melanoma cells by excessively inhibiting MCAM expression. Furthermore, miR-573 could suppress the growth of melanoma cells in vivo. These results highlight the role of miR-573 in melanoma and may provide insight to better understand the anticancer mechanism of miRNAs. 


\section{Materials and methods}

Cell lines and human tissues. Two melanoma cell lines, A375 and SK-MEL-2, were purchased from the American Type Culture Collection (Rockville, MD, USA). The cell lines were grown in RPMI-1640 medium containing $10 \%$ fetal bovine serum (FBS). Human melanoma specimens $(\mathrm{n}=11)$ and paired non-cancerous normal skin specimens $(n=11)$ were obtained from patients at the Affiliated Hospital of Medical College of Chinese People's Armed Police Forces, with documented informed consent for each case. Patients undergoing surgery for melanoma provided written consent to donate their tissues for analysis.

Quantitative PCR. The real-time PCR analyses for quantitation of miRNAs were carried out using SYBR ${ }^{\circledR}$ Premix Ex $\mathrm{Taq}^{\mathrm{TM}}$ II (Takara). After total RNA was extracted, miRNAspecific reverse transcription and PCR amplification were performed. The comparative cycle threshold $\left(\mathrm{C}_{t}\right)$ method was applied to quantify the expression levels through calculation using the $2^{-\Delta \Delta \mathrm{Ct}}$ method. U6 small nuclear RNA was used as an internal standard. The reverse transcription primers were: miR-573-RT, 5'-GTCGTATCCAGTGCAGGGTCCGAGGTG CACTGGATACGACCTGATC-3'; U6-RT, 5'-GTCGTATCC AGTGCAGGGTCCGAGGTGCACTGGATACGACAAAAT ATGG-3'. The PCR primers were: miR-573 forward, 5'-TGC GGCTGAAGTGATGTGTAAC-3'; U6 forward, 5'-TGCGG GTGCTCGCTTCGGCAGC-3' and a universal reverse primer, 5'-CCAGTGCAGGGTCCGAGGT-3'.

Cellular viability assay. A375 and SK-MEL-2 cells were transfected, trypsinized and seeded at $3 \times 10^{3}$ cells/well in 96-well plates. At $48 \mathrm{~h}$ after transfection, the 3-(4,5-dimethylthiazol-2-yl)-2,5-diphenyltetrazolium bromide, (MTT) assay was performed to determine the cellular viability. The optical density was read at $560 \mathrm{~nm}$ and the background was subtracted at $670 \mathrm{~nm}$.

In vitro invasion assay. Transwell invasion assays were performed to evaluate the invasion ability of the cells. Briefly, the transwell chambers were coated with $5 \mu \mathrm{g}$ of Matrigel (8 $\mu \mathrm{m}$ pore; BD Biosciences, Franklin Lakes, NJ, USA). The bottom chamber was filled with DMEM containing 10\% FBS. Tumor cells $\left(1 \times 10^{5}\right.$ cells in a total volume of $100 \mu 1$ FBS-free medium) were placed on the chamber and incubated at $37^{\circ} \mathrm{C}$ in $5 \% \mathrm{CO}_{2}$. Non-invading cells on the upper surface of the membrane were removed after $16 \mathrm{~h}$. The cells that invaded to the underside of the polycarbonate membrane were fixed with ethanol and stained by crystal violet for $5 \mathrm{~min}$. The number of invasive cells was then counted in five independent fields under a microscope. The mean of the triplicate assays for each experimental condition was used for analysis.

Apoptosis analysis. Flow cytometric analysis was performed using an Annexin V-PE/7-AAD apoptosis detection kit (BD Biosciences) to identify and quantify the apoptotic cells. Both adherent and floating cells were harvested and stained according to the manufacturer's instructions. The cell samples were analyzed using a Becton-Dickinson FACSVantage SE instrument.
Colony formation assay. Twenty-four hours after transfection, the cells $(1,000$ cells in a total volume of $2 \mathrm{ml})$ were placed on 6-well plates and incubated at $37^{\circ} \mathrm{C}$ in $5 \% \mathrm{CO}_{2}$ humidified air. After 15 days of incubation, the cells were stained by crystal violet and the number of clones was counted.

Vector construction. The miRNA expression plasmid was constructed using the expression plasmid pcDNA3.1. The DNA fragments containing miRNA precursor sequences were amplified from HEK293 cell genome using the following primers: pri-miR-573-F, 5'-CGCGGATCCACTTAAGGAGG GCTGAATGG-3' and pri-miR-573-R, 5'-CCGGAATTCAA CAGTGACTGTCCAAGAGC-3'; the PCR primers for MCAM 3'UTR fragment were: MCAM-3'UTR-F, 5'-AGCTTTG TTTAAACAATCACTTCAGCTCCCTTC-3' and MCAM3'UTR-R, 5'-CTAGTCTAGATGCAAATTTACACACCT GAC-3'. The fragment of mutated MCAM 3'UTR with mutated 3'UTR was amplified by PCR side-directed mutagenesis assay with these two primers (mutated MCAM-UTR-F and mutated MCAM-UTR-R). Mutated MCAM-UTR-F, 5'-AGCTTTGTT TAAACAAACTCATGACCTCCCTTC-3' and mutated MCAM-UTR-R, 5'-CTAGTCTAGATGCAAATTTACACA CCTGAC-3'. These two amplified fragments were both inserted into pmirGLO vector with $P m e \mathrm{I}$ and $\mathrm{Xba \textrm {I }}$ sites. The MCAM ectopic expression plasmid containing coding domains (CDS) was constructed using pCMV-HA vector. The PCR primers were: MCAM-F, 5'-CCGGAATTCATGGGG CTTCCCAGGCTGG-3' and MCAM-R, 5'-ATAAGAATG CGGCCGCCTAATGCCTCAGATCGATG-3'. The amplified fragment was inserted into pCMV-HA vector with EcoRI and NotI sites.

Luciferase assays. The luciferase reporter plasmids were transfected separately or cotransfected with miRNA expression vector into A375 cells using Lipofectamine 2000 (Invitrogen, Carlsbad, CA, USA) in 24-well plates. After $48 \mathrm{~h}$, the Firefly and Renilla luciferase activities were measured using the Dual-Glo ${ }^{\circledR}$ Luciferase Assay System (Promega, Madison, WI, USA) on Varioskan Flash microplate reader (Thermo Scientific) according to the manufacturer's protocols. The Firefly luciferase intensity was normalized to that of the Renilla luciferase. All the transfections were performed three times.

Western blot analysis. The cells were lysed in $0.4 \mathrm{ml}$ of lysis buffer (50 mM Tris pH 8.0,150 mM NaCl, 10 mM EDTA, $1 \%$ NP-40, $20 \mathrm{mM} \mathrm{NaF}, 1 \mathrm{mM}$ orthovanadate, and protease inhibitor cocktail). The lysates were separated by electrophoresis and transferred to the membrane, which was then blocked with bovine serum albumin. The membrane was then incubated with rabbit polyclonal antibody to MCAM (Abcam, Cambridge, UK) and then with goat anti-rabbit IgG-HRP secondary antibody (Santa Cruz Biotechnology, Inc., Santa Cruz, CA, USA). GAPDH (Santa Cruz) was used as loading control. Protein expression was assessed by enhanced chemiluminescence and exposure to the chemiluminescent film.

In vivo proliferation assay. The 5-week old $\mathrm{BABL} / \mathrm{c}$ nude mice were obtained from the Animal Center of the Military Academy of Medical Sciences (Beijing, China). All animal 

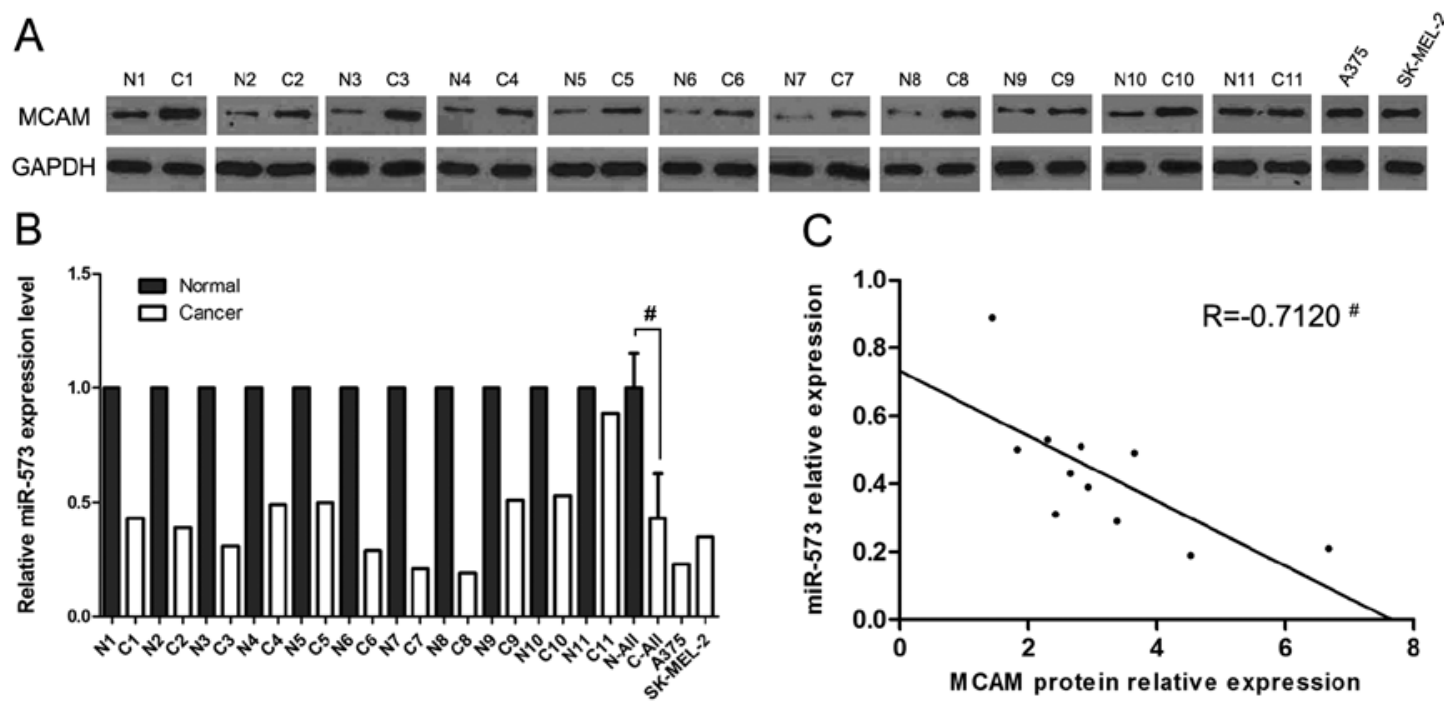

Figure 1. miR-573 is downregulated and inversely correlated with MCAM expression in melanoma. (A) MCAM protein levels in normal skin tissues (N), melanoma tissues (C) and two melanoma cell lines (A375 and SK-MEL-2) were detected using western blot assays, respectively. (B) The expression levels of miR-573 in N, C and two melanoma cell lines (A375 and SK-MEL-2) were measured using real-time PCR. U6 snRNA was used as loading control. (C) The correlation of MCAM and miR-573 levels $(\mathrm{D}, \mathrm{r}=-0.7120)$ is shown. $\left({ }^{*} \mathrm{P}<0.05\right)$.

A

MCAM 3'UTR

CDS

3'UTR

miR-573

5'- $\overline{N N N N U A G} \overline{C C C C G A A U C A C U U C A G}-3$

| | | | | | | | |

3'- $G A C \cup A G \cup C A A \cup G \cup G \cup A G \cup G A G \cup C$ -

1: | $1: 1: 11: 11$

Muted MCAM 3'UTR

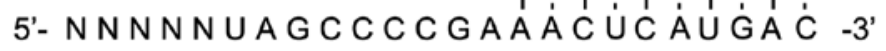

$\mathrm{B}$
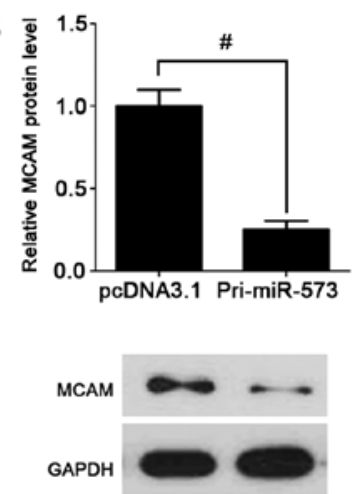

C

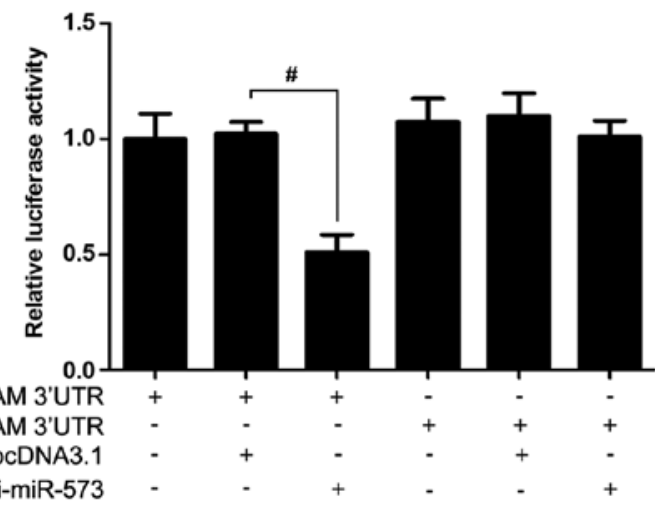

Figure 2. miR-573 inhibits the expression of MCAM by binding to 3'UTR. (A) Sequence alignment of miR-573 with the wild-type and mutant 3'UTR of MCAM. (B) The expression of MCAM in A375 cells transfected by miR-573 expression vector was tested by western blotting. (C) A375 cells were transfected with luciferase reporter vector along with the expression vectors of miR-573, miR-5095 or miR-4453. At $48 \mathrm{~h}$ after transfection, the Firefly and Renilla luciferase activities were measured. $\left({ }^{\#} \mathrm{P}<0.05\right)$.

studies were performed according to the animal use guidelines of the National Institute of Health and the current Chinese regulations and standards on the use of laboratory animals. A total of $1 \times 10^{7}$ cells were injected subcutaneously into nude mice. The mice were sacrificed at 30 days after injection. Tumor volume was evaluated using the following formula: Tumor volume $=4 \pi / 3 \times(\text { width } / 2)^{2} \times($ length $/ 2)$.

Statistical analysis. Data were expressed as mean \pm standard deviation (SD). Statistical comparisons were conducted between the two groups using the t-test. Differences were considered statistically significant when $\mathrm{P}<0.05$. The significant results are marked with (\#) in the figures.

\section{Results}

Expression levels of miR-573 are lower and are inversely correlated with MCAM expression in MCAM tissues. Real-time PCR methods were employed to compare the levels of miR-573 which were predicted to be potential direct regulators of MCAM by the TargetScan human database (release 6.2; http://www.targetscan.org) between 11 pairs of normal and matched human melanoma tissues. The levels of miR-573 in melanoma tissues and cell lines were lower than those in normal tissues (Fig. 1B). We also found that the MCAM protein expression levels were higher in melanoma tissues and cell lines compared to normal tissues (Fig. 1A). 
A

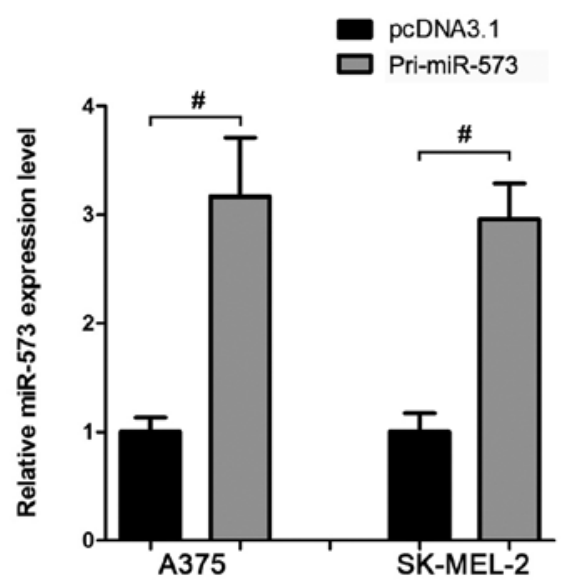

C
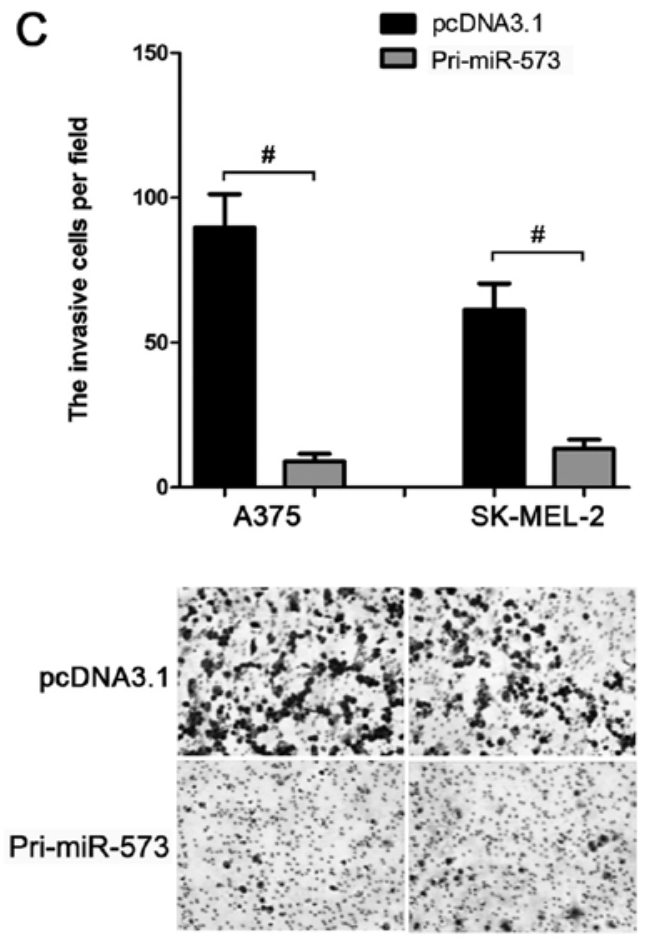

B
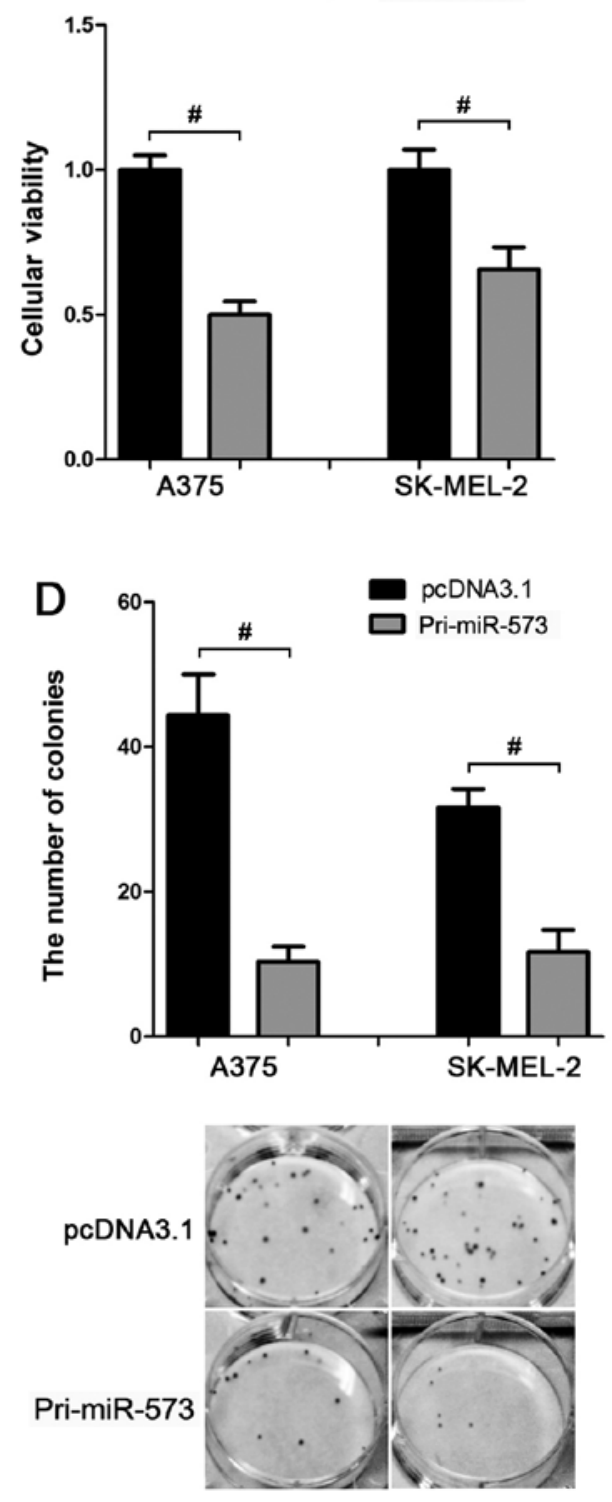

Figure 3. miR-573 promotes growth and invasion of A375 and SK-MEL-2 cells. (A) The miR-573 expression vector was transfected into A375 and SK-MEL-2 cells, and miR-573 level was measured using real-time PCR. (B) miR-573 was overexpressed in A375 and SK-MEL-2 cells, and the cellular viability was detected using MTT assay. (C) miR-573 was overexpressed in A375 and SK-MEL-2 cells, and the cell invasion activity was detected using transwell assay. (D) miR-573 was overexpressed in A375 and SK-MEL-2 cells, and the cellular colony formation activity was detected. ( $\left.{ }^{\#} \mathrm{P}<0.05\right)$.

miR-573 directly regulates the expression of MCAM. To further investigate which miRNA influenced MCAM expression, miR-573 was overexpressed in A375 cells. Compared to the control group, MCAM expression in miR-573 overexpressed cells was lower (Fig. 2B). Furthermore, luciferase reporter assay showed that the transfection of miR-573 expression vector significantly suppressed the luciferase intensity with miR-573 binding sites (Fig. 2C). These data support the hypothesis that miR-573 is a direct regulator of MCAM.

miR-573 inhibits the proliferation and invasion of melanoma cells. Based on the above data, we inferred that miR-573 was downregulated in melanoma cells. This led us to evaluate whether miR-573 exhibited a tumor-suppressive function in melanoma development. MTT assay showed that compared to the control group, transfection of miR-573 expression vector (Fig. 3A) suppressed the cellular viability by $\sim 40-50 \%$ in A375 and SK-MEL-2 cells (Fig. 3B). In addition, the invasion (Fig. 3C) and colony formation (Fig. 3D) activities of these two cell lines were also inhibited when miR-573 was overexpressed. Thus, miR-573 may play a tumor-suppressive role in melanoma cells.

Overexpression of MCAM prevents the negative effects of miR-573 on melanoma cells. To investigate whether miR-573 suppressed melanoma progression through directly and negatively regulating MCAM, an MCAM ectopic expression vector was introduced (Fig. 4A) to weaken or eliminate the negative effects of miR-573 on melanoma cells. As a result, subsequent transfection of MCAM ectopic expression vector into the 

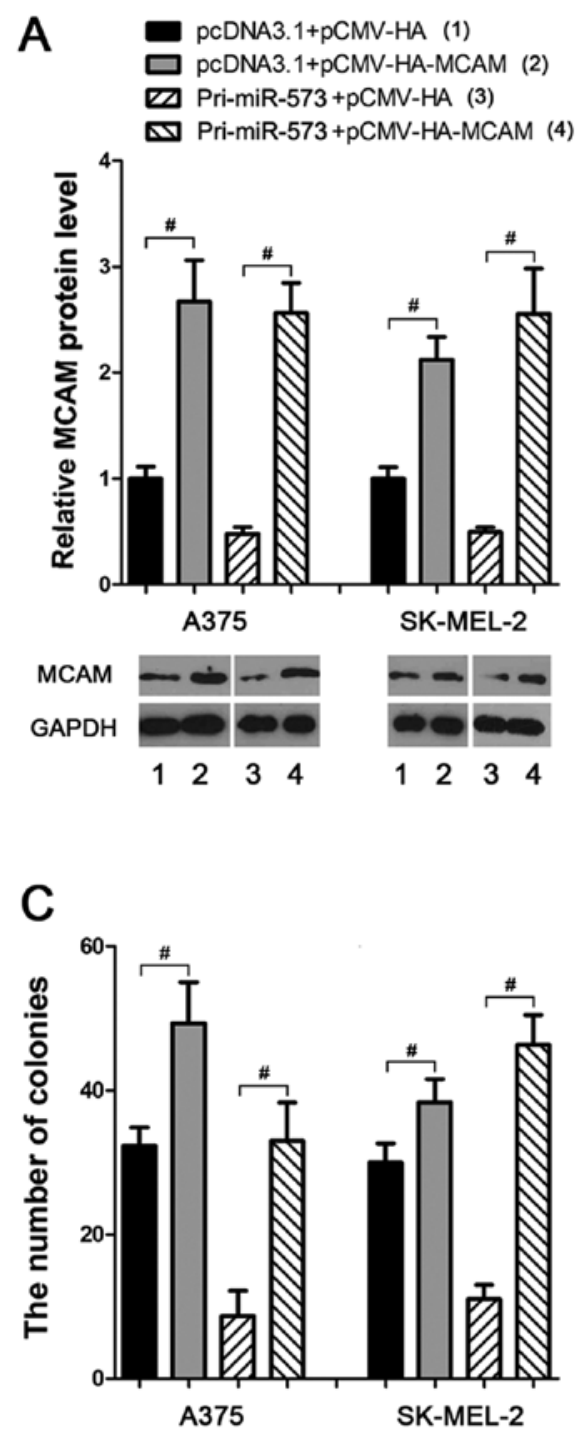

D

1 pcDNA3.1+pCMV-HA

2 pcDNA3.1+pCMV-HA-MCAM
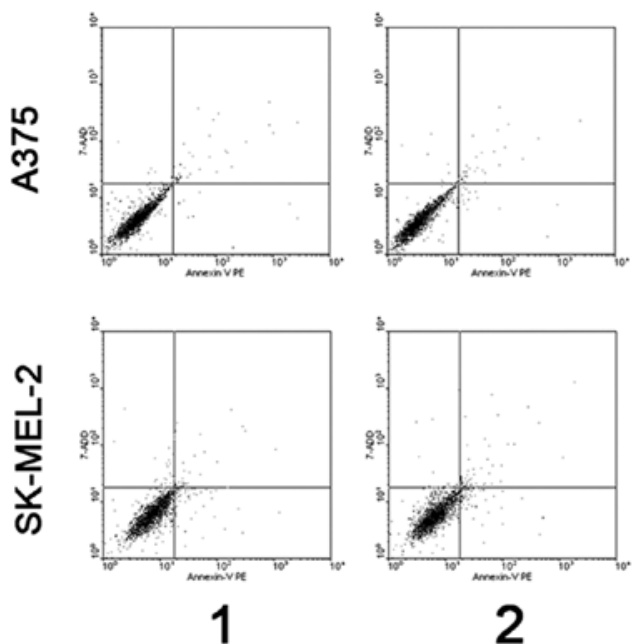
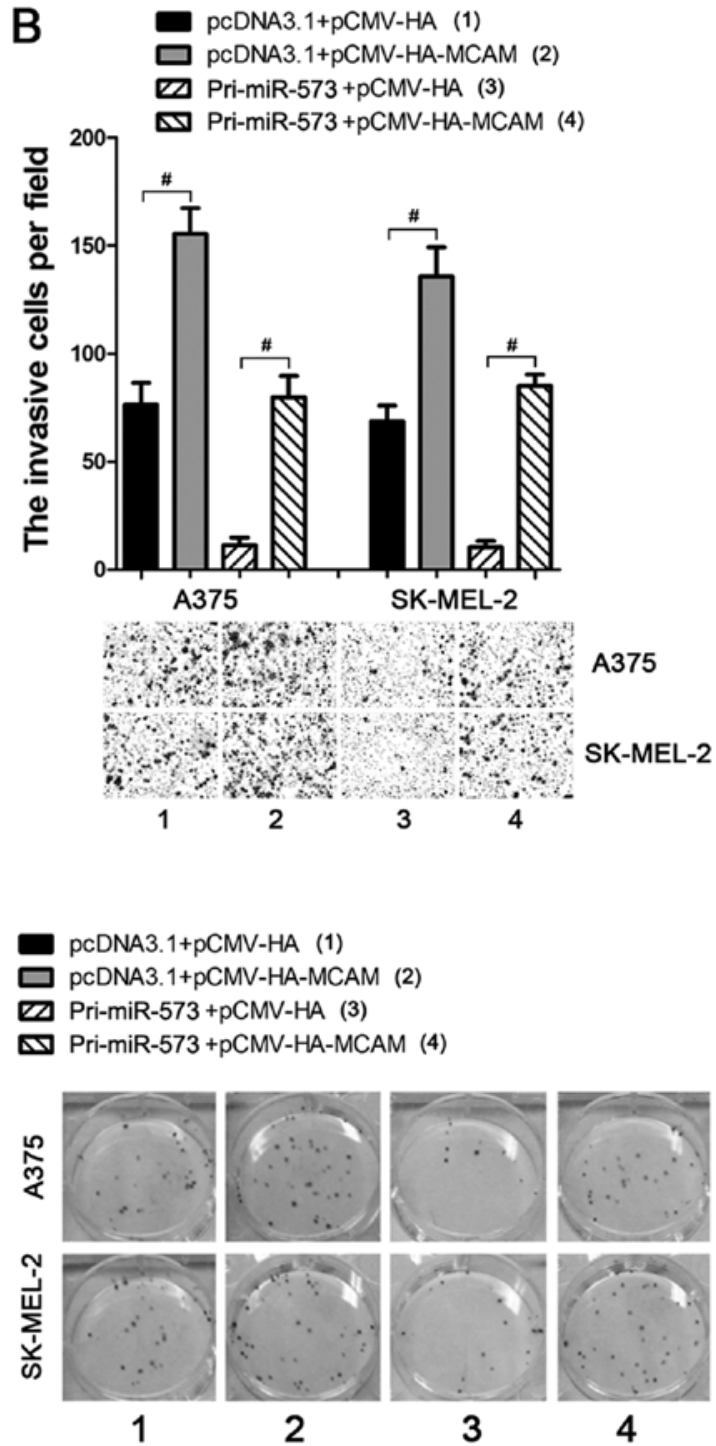

3 Pri-miR-573+pCMV-HA

4 Pri-miR-573+pCMV-HA-MCAM
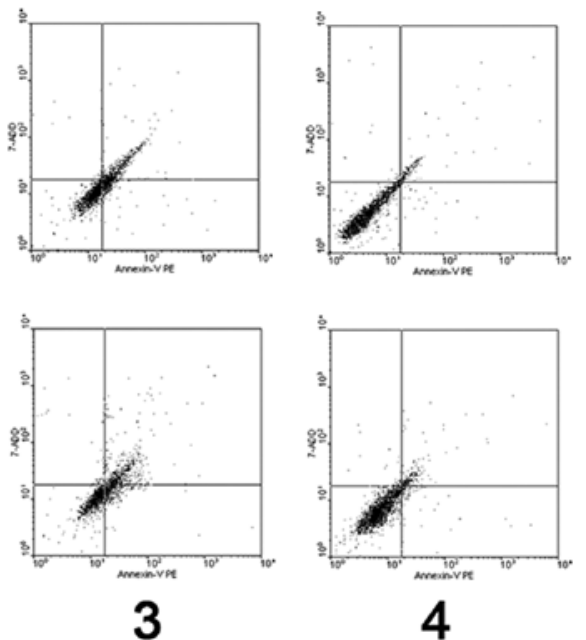

Figure 4. miR-573 suppresses the phenotypes of melanoma cells via negatively regulating MCAM. (A) A375 and SK-MEL-2 cells were transfected with miR-573 expression vector and subsequently with MCAM expression vector. The MCAM protein level was measured using western blot assay. (B) Melanoma cells were treated as described above, and the cell invasion activity was detected using transwell assay. (C) Melanoma cells were treated as described above, and the cellular colony formation activity was detected. (D) Melanoma cells were treated as described above, and cell apoptosis was measured using Annexin V-PE based staining and flow cytometry analyses. $\left({ }^{\#} \mathrm{P}<0.05\right)$. 

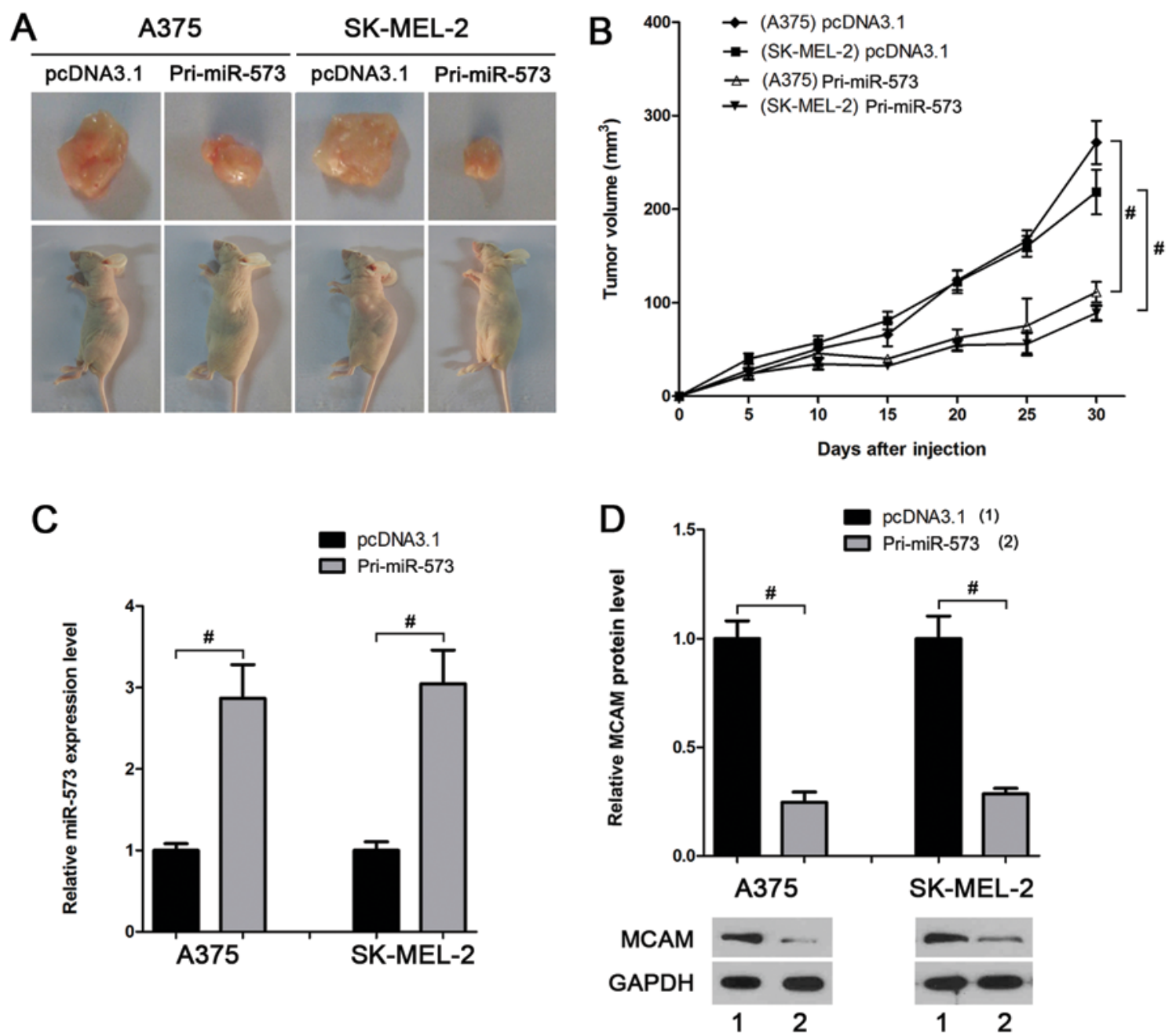

Figure 5. miR-573 suppresses melanoma cell derived allogeneic tumors in vivo. The melanoma cells stably expressing miR-573 were injected subcutaneously into nude mice. At 30 days after injection, (A) the mice were sacrificed, the tumors were isolated, and (B) the tumor volume was measured. (C) miR-573 level in the allogeneic neoplasms was measured using real-time PCR. (D) MCAM protein level in the allogeneic neoplasms was measured using western blot assay. $\left({ }^{\#} \mathrm{P}<0.05\right)$.

miR-573 treated A375 and SK-MEL-2 cells led to enhancement of the cellular invasion (Fig. 4B) and colony formation (Fig. 4C) activities. Moreover, using the Annexin V-PE based apoptosis analysis, miR-573 enhanced apoptosis of melanoma cells, which could also be eliminated by subsequent overexpression of MCAM (Fig. 4D). These data provide strong evidence that miR-573 exhibits tumor-suppressive activities through negatively regulating MCAM expression in melanoma cells.

miR-573 inhibits melanoma growth in vivo. The melanoma cell lines that stably express miR-573, A375-miR-573 and SK-MEL-2-miR-573, were established to further investigate the effect of miR-573 on melanoma cells in vivo. The volume of allogeneic tumor in mice injected with cells overexpressing miR-573 was smaller than that in the control groups (Fig. 5A and B). Real-time PCR and western blot analyses suggested a higher miR-573 level (Fig. 5C) and a lower MCAM protein level (Fig. 5D) in the tumors derived from A375-miR-573 or SK-MEL-2-miR-573, further suggesting that miR-573 suppresses melanoma development in vivo by inhibiting MCAM.

\section{Discussion}

Malignant melanoma remains the most aggressive form of skin cancer. The data obtained from the World Health Organization (WHO) showed that the number of melanoma cases worldwide is increasing faster than any other type of cancer (11). Extensive efforts focusing on miRNAs have been made to elucidate the molecular mechanisms underlying the malignant behavior of transformed melanocytes (12-15). To date, miRNAs have been developed as potential therapeutic targets. For example, miR-221/-222 cluster is a promising target in more advanced melanomas. miRNAs of the let-7 family, particularly let-7a, may also be of interest in early melanoma stages. Moreover, miR-21, miR-205 and miR-34a are also reported to regulate melanoma cell progression (16-18).

In the present study, we first compared the expression of miR-573 that was algorithmically predicted to be a potential upstream regulator of MCAM. The results showed that miR-573 in melanoma tissues was lower than that in normal tissues. miR-573 also exhibited low expression level in the two typical melanoma cell lines, A375 and SK-MEL-2. 
MCAM, a cell-surface glycoprotein that mediates heterotypic and homeotypic cell-cell adhesion, exhibits abnormal expression in a variety of tumors $(5,19,20)$. For example, overexpressed MCAM in melanoma cells significantly promotes the growth and metastasis of tumors in nude mice (21). Therefore, we further examined the miRNAs that may directly regulate MCAM expression. The results showed that the MCAM mRNA and protein levels were higher in melanoma tissues and cell lines, which were clearly regulated by the miRNA miR-573. The growth and invasion abilities of melanoma cells were significantly reduced by miR-573. In addition, overexpression of MCAM can inhibit the effect of miR-573 on cellular malignant phenotypes. The in vivo results also demonstrated that the volume of allogeneic tumors was significantly reduced when miR-573 was overexpressed in the melanoma cells.

To date, the diagnosis for melanoma is poor and efficient therapeutic methods are lacking. The results obtained from the present study may extend our knowledge of melanoma progression and facilitate the establishment of miRNA-based therapies.

\section{References}

1. Ferlay J, Autier P, Boniol M, Heanue M, Colombet M and Boyle P: Estimates of the cancer incidence and mortality in Europe in 2006. Ann Oncol 18: 581-592, 2007.

2. Lehmann JM, Riethmuller G and Johnson JP: MUC18, a marker of tumor progression in human melanoma, shows sequence similarity to the neural cell adhesion molecules of the immunoglobulin superfamily. Proc Natl Acad Sci USA 86: 9891-9895, 1989.

3. Wu GJ, Peng Q, Fu P, et al: Ectopical expression of human MUC18 increases metastasis of human prostate cancer cells. Gene 327: 201-213, 2004

4. Zabouo G, Imbert AM, Jacquemier J, et al: CD146 expression is associated with a poor prognosis in human breast tumors and with enhanced motility in breast cancer cell lines. Breast Cancer Res 11: R1, 2009.

5. Kristiansen G, Yu Y, Schlüns K, Sers C, Dietel M and Petersen I: Expression of the cell adhesion molecule CD146/MCAM in non-small cell lung cancer. Anal Cell Pathol 25: 77-81, 2003.
6. Bartel DP: MicroRNAs: target recognition and regulatory functions. Cell 136: 215-233, 2009.

7. Filipowicz W, Bhattacharyya SN and Sonenberg N: Mechanisms of post-transcriptional regulation by microRNAs: are the answers in sight? Nat Rev Genet 9: 102-114, 2008.

8. Kim VN, Han J and Siomi MC: Biogenesis of small RNAs in animals. Nat Rev Mol Cell Biol 10: 126-139, 2009.

9. Carthew RW and Sontheimer EJ: Origins and mechanisms of miRNAs and siRNAs. Cell 136: 642-655, 2009.

10. Glud M and Gniadecki R: MicroRNAs in the pathogenesis of malignant melanoma. J Eur Acad Dermatol Venereol 27: 142-150, 2013.

11. Parkin DM, Bray F, Ferlay $\mathbf{J}$ and Pisani P: Global cancer statistics, 2002. CA Cancer J Clin 55: 74-108, 2005.

12. Mueller DW and Bosserhoff AK: Role of miRNAs in the progression of malignant melanoma. Br J Cancer 101: 551-556, 2009.

13. Mueller DW, Rehli M and Bosserhoff AK: miRNA expression profiling in melanocytes and melanoma cell lines reveals miRNAs associated with formation and progression of malignant melanoma. J Invest Dermatol 129: 1740-1751, 2009.

14. Noguchi S, Mori T, Otsuka Y, et al: Anti-oncogenic microRNA203 induces senescence by targeting E2F3 protein in human melanoma cells. J Biol Chem 287: 11769-11777, 2012.

15. Essa S, Reichrath S, Mahlknecht U, Montenarh M, Vogt T and Reichrath J: Signature of VDR miRNAs and epigenetic modulation of vitamin D signaling in melanoma cell lines. Anticancer Res 32: 383-389, 2012.

16. Yang CH, Yue J, Pfeffer SR, Handorf CR and Pfeffer LM: MicroRNA miR-21 regulates the metastatic behavior of B16 melanoma cells. J Biol Chem 286: 39172-39178, 2011.

17. Hanna JA, Hahn L, Agarwal S and Rimm DL: In situ measurement of miR-205 in malignant melanoma tissue supports its role as a tumor suppressor microRNA. Lab Invest 92: 1390-1397, 2012.

18. Cozzolino AM, Pedace L, Castori M, et al: Analysis of the miR-34a locus in 62 patients with familial cutaneous melanoma negative for CDKN2A/CDK4 screening. Fam Cancer 11: 201-208, 2012.

19. Wu Z, Wu Z, Li J, Yang X, et al: MCAM is a novel metastasis marker and regulates spreading, apoptosis and invasion of ovarian cancer cells. Tumour Biol 33: 1619-1628, 2012.

20. Liu JW, Nagpal JK, Jeronimo C, et al: Hypermethylation of MCAM gene is associated with advanced tumor stage in prostate cancer. Prostate 68: 418-426, 2008

21. Wu GJ, Fu P, Wang SW and Wu MW: Enforced expression of MCAM/MUC18 increases in vitro motility and invasiveness and in vivo metastasis of two mouse melanoma K1735 sublines in a syngeneic mouse model. Mol Cancer Res 6: 1666-1677, 2008. 